UTHEP-669

\title{
Matrix Geometry and Coherent States
}

\author{
Goro IsHikl* \\ Graduate School of Pure and Applied Sciences, University of Tsukuba, \\ Tsukuba, Ibaraki 305-8571, Japan \\ Yukawa Institute for Theoretical Physics, Kyoto University \\ Kyoto, 606-8502, Japan
}

\begin{abstract}
We propose a novel method of finding the classical limit of the matrix geometry. We define coherent states for a general matrix geometry described by a large- $N$ sequence of $D$ Hermitian matrices $X_{\mu}(\mu=1,2, \cdots, D)$ and construct a corresponding classical space as a set of all coherent states. When the classical space forms a smooth manifold, we also express various geometric objects on the classical space such as the metric, Levi-Civita connection, curvature and Poisson tensor, in terms of the matrix elements. This method provides a new class of observables in matrix models, which characterize geometric properties of matrix configurations.
\end{abstract}

\footnotetext{
* e-mail address : ishiki@het.ph.tsukuba.ac.jp
} 


\section{Introduction}

Matrix models are conjectured to give nonperturbative formulations of string and M theories [1, 2]. In the matrix models, classical Riemannian geometry is replaced by a kind of quantum geometry described by matrices [3] and the matrix models are expected to realize a novel description of gravitational theories based on the matrix geometry. However, the relation between the matrix geometry and Riemannian geometry has not been fully understood so far 1. Finding a clear relation between these two geometries, which look quite different at first sight, will help to understand the matrix models as theories of gravity.

Although concrete examples of the matrix geometry (the matrix regularization) have been constructed for some manifolds [5 9], any algorithmic construction method has not been known for a general manifold. Also, its inverse problem, namely the problem of finding an associated classical (commutative) manifold for a given matrix geometry, has been only partly understood. For the latter problem, the Morse theoretic method [10] and the method of taking an optimal gauge [11,12] have been proposed. These are useful to see some geometric aspects such as the topology or the shape of the classical space for a given matrix configuration. They are, however, not convenient enough to see more detailed information such as relations between matrix configurations and geometric objects on the classical space such as the curvature tensors. For the case of two-dimensional surfaces, matrices which correspond to some geometric objects were explicitly constructed in [13. Recently, another method of finding the classical space using a Dirac operator was proposed in [14,15]. This method is closely related to our proposal.

In this paper, we propose a novel approach to the latter problem by generalizing the notion of coherent states. Coherent states are often considered for the Heisenberg algebra $[\hat{p}, \hat{q}]=-i \hbar$ (see Appendix $[\mathrm{A}$ ) and play an important role in taking the classical limit. They are approximate simultaneous eigenstates of $\hat{p}$ and $\hat{q}$ and can be defined as the states which saturate the uncertainty bound. They are considered as the quantum analogue of the points on the classical phase space. Indeed, there exists a one-to-one correspondence between the classical plane and a set of all coherent states. This means that, from the Heisenberg algebra, one can construct the classical geometry as a set of all coherent states.

\footnotetext{
${ }^{1}$ For infinitely large matrices, a very interesting solution to this problem was found in 4 .
} 
The same construction is possible for the case of the fuzzy sphere, where the Bloch (spin) coherent states are in one-to-one correspondence with the points on $S^{2}$ (see Appendix B). In this paper, we extend this construction to more general matrix geometries.

For a Poisson manifold $\mathcal{M}$, its matrix regularization can be defined in terms of a large$N$ sequence of matrices (for example, see [13]). Let $T_{N}$ be a linear map from $C^{\infty}(\mathcal{M})$ to a set of all $N \times N$ matrices. When the sequence $\left\{T_{N}\right\}_{N}$, where $N$ is monotonically increasing to infinity, satisfies the four conditions shown in Eqs. (4.1)-(4.4) in [13], $\left\{T_{N}\right\}_{N}$ is called the matrix regularization of $\mathcal{M}$. The main property of the matrix regularization is the first two of the four, which take the following form:

$$
\begin{aligned}
& \lim _{N \rightarrow \infty}\left\|T_{N}(f) T_{N}(g)-T_{N}(f g)\right\|=0, \\
& \lim _{N \rightarrow \infty}\left\|i N\left[T_{N}(f), T_{N}(g)\right]-T_{N}(\{f, g\})\right\|=0 .
\end{aligned}
$$

These conditions say that the map preserves the algebraic structure of functions and the Poisson bracket is well approximated by the commutators of matrices. In this paper, we consider the case where the manifold $\mathcal{M}$ is embedded in a flat space. For the embedding function $y^{\mu}: \mathcal{M} \rightarrow \mathbf{R}^{D}$, we denote by $X^{\mu}=T_{N}\left(y^{\mu}\right)$ the images of $y^{\mu}$. Then, the first condition in (1.1) means that the polynomials $y^{\mu} y^{\nu} \cdots$ are mapped to the matrix polynomials $X^{\mu} X^{\nu} \cdots$ up to the $1 / N$ corrections, while the second means that

$$
i N\left[X^{\mu}, X^{\nu}\right]-W^{\mu \nu}(X) \rightarrow 0
$$

where $W^{\mu \nu}$ is the induced Poisson tensor2. Thus, the matrix regularization for an embedded space is given by a large- $N$ sequence of Hermitian matrices $\left\{X^{\mu}\right\}_{N}$ which satisfies (1.2). See [9] for more detailed description for the embedded case. In the matrix regularization of string and $\mathrm{M}$ theories, the matrices $X^{\mu}$ are treated as the dynamical degrees of freedom and considered to realize a matrix regularization of the worldsheet of strings or the transverse worldvolume of membranes.

In this paper, we will consider the inverse problem of the construction of the matrix regularization. Namely, we start from a large- $N$ sequence of (bounded) matrices $\left\{X^{\mu}\right\}_{N}$, which may or may not realize a matrix regularization of a certain manifold, and consider

\footnotetext{
${ }^{2}$ Of course, there is an ordering ambiguity in defining $W^{\mu \nu}$ with the matrix argument. However, (1.2) also implies that $X^{\mu}$ commute with each other in the large- $N$ limit so that the ambiguity is negligibly small.
} 
how a classical space $\mathcal{M}$ can be associated with the matrix sequence. This kind of problem is relevant for some physically interesting situations, such as the emergent geometry in the gauge/gravity correspondence and the matrix realization of membranes. For this problem, (1.1) is not very useful since at the beginning we do not know what the space $\mathcal{M}$ should be. Instead, we define coherent states for a given sequence $\left\{X^{\mu}\right\}_{N}$ as a generalization of the known cases and then define the classical space $\mathcal{M}$ as a set of all the coherent states.

For the cases where the classical space defined in this way forms a smooth manifold, we also express geometric objects on the classical space such as the metric, Levi-Civita connection, curvature and Poisson tensor, in terms of the matrix elements. If we consider a completely generic configuration of matrices, the classical space of our definition could be nonmanifold or an empty set. So in this sense, our result on the geometric objects cannot be applied to a generic matrix configuration whose classical geometries are not smooth manifolds. However, as will be discussed in the last section, our method is still valid for a small perturbation around matrices which have smooth classical geometry.

The organization of this paper is as follows. In Sec. 2, we introduce the notion of coherent states for a general matrix geometry and define a classical space as a set of all coherent states. In Sec. 3, we express the metric, connection, curvature and Poisson tensor

on the classical space in terms of matrix elements. In Sec. 4, we show some examples of our construction. In Sec. 5, we consider the case of infinite-dimensional matrices. In Sec. 6. we summarize our result and discuss possible applications.

\section{Classical geometry of finite size matrices}

In this section, we consider the classical (commutative) limit of finite size matrices. We denote an index set by $I:=\left\{a_{n} \mid n \in \mathbf{N}\right\}$, where $\left\{a_{n}\right\}$ is a strictly monotonically increasing sequence of natural numbers. We assume that we are given a family of $D$ matrices,

$$
\left\{\left(X_{1}^{(N)}, X_{2}^{(N)}, \cdots, X_{D}^{(N)}\right) \mid N \in I\right\}
$$

where $X_{\mu}^{(N)}(\mu=1,2, \cdots, D)$ are $N \times N$ Hermitian matrices. We consider $X_{\mu}^{(N)}$ as regularized embedding functions with the target space $\mathbf{R}^{D}$, which possibly realize a matrix regularization of a certain manifold. We raise and lower the $D$-dimensional indices by 
using the Kronecker delta (the flat metric on $\mathbf{R}^{D}$ ), so that we do not distinguish upper and lower indices in the following.

Let us define the position and its standard deviation in $\mathbf{R}^{D}$ for each state 3 . Let $\mathcal{H}$ be the $N$-dimensional Hilbert space on which the $D$ matrices act and $\mathcal{H}^{*}$ be a subset of $\mathcal{H}$ which consists of normalized state vectors:

$$
\mathcal{H}^{*}=\{|\alpha\rangle \in \mathcal{H} \mid\langle\alpha \mid \alpha\rangle=1\}
$$

For each $|\alpha\rangle \in \mathcal{H}^{*}$, we define the position of $|\alpha\rangle$ in $\mathbf{R}^{D}$ by

$$
x_{\mu}(|\alpha\rangle)=\left\langle\alpha\left|X_{\mu}\right| \alpha\right\rangle \quad(\mu=1,2, \cdots, D)
$$

and the standard deviations by

$$
\begin{aligned}
\sigma_{\mu}^{2}(|\alpha\rangle) & =\left\langle\alpha\left|X_{\mu}^{2}\right| \alpha\right\rangle-\left\langle\alpha\left|X_{\mu}\right| \alpha\right\rangle^{2}(\mu=1,2, \cdots, D), \\
\sigma^{2}(|\alpha\rangle) & =\sum_{\mu=1}^{D} \sigma_{\mu}^{2}(|\alpha\rangle) .
\end{aligned}
$$

Next, we introduce the notion of the coherent states. We recall that the canonical coherent states can be defined as the ground states of the Hamiltonians, $H\left(p_{0}, q_{0}\right)=$ $\frac{1}{2}\left(\hat{p}-p_{0}\right)^{2}+\frac{1}{2}\left(\hat{q}-q_{0}\right)^{2}$, where $p_{0}$ and $q_{0}$ are real parameters (see Appendix $\mathrm{A}$ ). We generalize this definition. We first introduce the "Hamiltonian"

$$
H(y)=\frac{1}{2} \sum_{\mu=1}^{D}\left(X_{\mu}-y_{\mu}\right)^{2}
$$

This is an $N \times N$ Hermitian matrix defined for each point $y \in \mathbf{R}^{D}$. The $N \times N$ identity matrix is omitted in the term of $y_{\mu}$. We denote the $n$th eigenstate and the eigenvalue of $H(y)$ by $|n, y\rangle$ and $E_{n}(y)$ :

$$
H(y)|n, y\rangle=E_{n}(y)|n, y\rangle \quad(n=0,1, \cdots, N-1)
$$

where we assume that the eigenvalues are ordered as $E_{0}(y) \leq E_{1}(y) \leq \cdots \leq E_{N-1}(y)$ and the states are normalized as $\langle n, y \mid m, y\rangle=\delta_{m n}$. Our definition of coherent states is the

\footnotetext{
${ }^{3}$ In the following, we omit the superscript of $(N)$ and make the $N$-dependence implicit to avoid complexity.
} 
following. We call $|0, y\rangle$ a coherent state at $y$ if and only if it satisfies 4

$$
\lim _{N \rightarrow \infty} E_{0}(y)=0
$$

Our definition of coherent states can be understood as follows. Suppose that the coherent state exists at a point $y \in \mathbf{R}^{D}$. Since the ground state energy can be written as

$$
E_{0}(y)=\frac{1}{2} \sigma^{2}(|0, y\rangle)+\frac{1}{2} \sum_{\mu=1}^{D}\left(x_{\mu}(|0, y\rangle)-y_{\mu}\right)^{2},
$$

Eq. (2.7) implies that $\sigma(|0, y\rangle) \rightarrow 0$ and $x_{\mu}(|0, y\rangle) \rightarrow y_{\mu}$. Thus, it follows that there exists a state whose wave packet is centered at $y$ and shrinks to the point in the large- $N$ limit. Conversely, suppose that there exists a state $|\alpha\rangle \in \mathcal{H}^{*}$ which satisfies $\sigma(|\alpha\rangle) \rightarrow 0$ and $x_{\mu}(|\alpha\rangle) \rightarrow y_{\mu}$. Then, $\langle\alpha|H(y)| \alpha\rangle$ goes to zero in the large- $N$ limit. Since $E_{0}(y)$ can be written as

$$
E_{0}(y)=\min _{|\psi\rangle \in \mathcal{H}^{*}}\langle\psi|H(y)| \psi\rangle
$$

it also goes to zero in the large- $N$ limit; namely, a coherent state exists at $y$. Therefore, for each $y \in \mathbf{R}^{D}$, if and only if a coherent state exists, there exists a wave packet which shrinks to a point in the large- $N$ limit.

It is natural to define the classical space, which we denote by $\mathcal{M}$, as a subspace of $\mathbf{R}^{D}$ on which there exist the shrinking wave functions. In terms of the coherent states, this is equivalent to the subspace of $\mathbf{R}^{D}$ on which there exist the coherent states. Let us define a function $f: \mathbf{R}^{D} \rightarrow \mathbf{R}_{+}$as

$$
f(y)=\lim _{N \rightarrow \infty} E_{0}(y)
$$

Then, we can write $\mathcal{M}$ as

$$
\mathcal{M}=\left\{y \in \mathbf{R}^{D} \mid f(y)=0\right\}
$$

Note that one can compute $f(y)$ from the given matrices in principle. So the expression (2.11) provides a relation between the classical space and the matrix configurations.

\footnotetext{
${ }^{4}$ Note that strictly speaking the coherent state defined here is not a single state vector but a set of the ground states which satisfy (2.7).
} 
Let us comment on some properties of the function $f(y)$. Suppose that $E_{0}(y)$ is a smooth function at a point $y \in \mathbf{R}^{D}$, we can expand $E_{0}(y+\epsilon)$ in a Taylor series as

$$
E_{0}(y+\epsilon)=E_{0}(y)+\epsilon^{\mu} \partial_{\mu} E_{0}(y)+\frac{1}{2} \epsilon^{\mu} \epsilon^{\nu} \partial_{\mu} \partial_{\nu} E_{0}(y)+\frac{1}{6} \epsilon^{\mu} \epsilon^{\nu} \epsilon^{\rho} \partial_{\mu} \partial_{\nu} \partial_{\rho} E_{0}(y)+\cdots
$$

Since $E_{0}(y+\epsilon)$ is the lowest eigenvalue of

$$
H(y+\epsilon)=H(y)+\epsilon_{\mu}\left(y^{\mu}-X^{\mu}\right)+\frac{1}{2}\left(\epsilon^{\mu}\right)^{2},
$$

we can compute the coefficients in (2.12) based on the perturbation theory a 5

$$
\begin{aligned}
\partial_{\mu} E_{0}(y) & =y_{\mu}-x_{\mu}(|0, y\rangle), \\
\partial_{\mu} \partial_{\nu} E_{0}(y)= & \delta_{\mu \nu}-2 \sum_{n=1}^{N-1} \operatorname{Re} \frac{\left\langle 0, y\left|X_{\mu}\right| n, y\right\rangle\left\langle n, y\left|X_{\nu}\right| 0, y\right\rangle}{E_{n}(y)-E_{0}(y)}, \\
\partial_{\mu} \partial_{\nu} \partial_{\rho} E_{0}(y)= & \sum_{m \neq 0} \sum_{n \neq 0} \frac{\left\langle 0, y\left|X_{\mu}\right| m, y\right\rangle\left\langle m, y\left|X_{\nu}\right| n, y\right\rangle\left\langle n, y\left|X_{\rho}\right| 0, y\right\rangle}{\left(E_{m}(y)-E_{0}(y)\right)\left(E_{n}(y)-E_{0}(y)\right)} \\
& -\left\langle 0, y\left|X_{\mu}\right| 0, y\right\rangle \sum_{n \neq 0} \frac{\left\langle 0, y\left|X_{\nu}\right| n, y\right\rangle\left\langle n, y\left|X_{\rho}\right| 0, y\right\rangle}{\left(E_{n}(y)-E_{0}(y)\right)^{2}}+\cdots,
\end{aligned}
$$

where $\cdots$ represents the sum over all permutations of the indices $\mu, \nu, \rho$. We can also expand the function $f(y)$ as

$$
f(y+\epsilon)=f(y)+\epsilon^{\mu} \partial_{\mu} f(y)+\frac{1}{2} \epsilon^{\mu} \epsilon^{\nu} \partial_{\mu} \partial_{\nu} f(y)+\frac{1}{6} \epsilon^{\mu} \epsilon^{\nu} \epsilon^{\rho} \partial_{\mu} \partial_{\nu} \partial_{\rho} f(y)+\cdots,
$$

where $\partial_{\mu} f(y), \partial_{\mu} \partial_{\nu} f(y)$ and $\partial_{\mu} \partial_{\nu} \partial_{\rho} f(y)$ are given by the large- $N$ limits of (2.14). Thus, we can compute the derivatives of $f(y)$ as the corrections in the perturbation theory. This property plays an important role, when we define geometric objects in terms of the matrix elements in the next section. Note also that since $x^{\mu}(|0, y\rangle)$ goes to $y^{\mu}$ for $y \in \mathcal{M}$ as discussed above, the single derivative of $f(y)$ is vanishing on $\mathcal{M}$ :

$$
\partial_{\mu} f(y)=0(\mu=1,2, \cdots, D, y \in \mathcal{M})
$$

Hence, $f(y)$ is a quadratic function on a neighborhood of $\mathcal{M}$.

\footnotetext{
${ }^{5}$ Here, we assume that the Hamiltonian is nondegenerate, but we can also treat degenerate cases in a similar way.
} 
The coherent state at $y$ is an approximate simultaneous eigenstate of the $D$ matrices, where the eigenvalues are given by the components of $y$. In fact, it follows from (2.7) and the Cauchy-Schwarz inequality that, for any vector $|\psi\rangle \in \mathcal{H}^{*}$,

$$
\lim _{N \rightarrow \infty}\left\langle\psi\left|X^{\mu}-y^{\mu}\right| 0, y\right\rangle=0 \quad(\mu=1,2, \cdots, D, \quad y \in \mathcal{M}) .
$$

From (2.17), one can also show the following equations for $y \in \mathcal{M}$ and for any state vector $|\psi\rangle \in \mathcal{H}^{*}:$

$$
\begin{aligned}
& \lim _{N \rightarrow \infty}\langle\psi|A(X-y)| 0, y\rangle=0, \\
& \lim _{N \rightarrow \infty}(\langle\psi|A(X)| 0, y\rangle-\langle\psi \mid 0, y\rangle\langle 0, y|A(X)| 0, y\rangle)=0, \\
& \lim _{N \rightarrow \infty}\langle\psi|[A(X), B(X)]| 0, y\rangle=0,
\end{aligned}
$$

where $A(X)$ and $B(X)$ are arbitrary polynomials of $X^{\mu}$ with finite ( $N$-independent) degrees and coefficients. Here, we have assumed that $X^{\mu}$ are bounded in the large- $N$ limit.

\section{$3 \quad$ Geometric objects}

In the following, we assume that there exists a neighborhood of $\mathcal{M}$ on which $E_{0}(y)$ is smooth 6 for any sufficiently large $N$. In this case, the classical space $\mathcal{M}$ is a smooth submanifold of $\mathbf{R}^{D}$. In this section, under this assumption, we define various geometric objects such as the metric, Levi-Civita connection, curvature, and Poisson tensor on $\mathcal{M}$, in terms of the $D$ matrices $X^{\mu}$. In the following, we assume for simplicity that the Hamiltonian (2.5) is nondegenerate on the neighborhood of $\mathcal{M}$, but the generalization to degenerate cases is straightforward.

\subsection{Metric}

We consider a real symmetric $D \times D$ matrix

$$
g_{\mu \nu}(y)=2 \lim _{N \rightarrow \infty} \sum_{n=1}^{N-1} \operatorname{Re} \frac{\left\langle 0, y\left|X_{\mu}\right| n, y\right\rangle\left\langle n, y\left|X_{\nu}\right| 0, y\right\rangle}{E_{n}(y)-E_{0}(y)} .
$$

\footnotetext{
${ }^{6}$ In order for the curvature tensors to be defined, $E_{0}(y)$ needs to be at least 3 times differentiable on the neighborhood.
} 
Note that this can also be written as

$$
g_{\mu \nu}(y)=\delta_{\mu \nu}-\partial_{\mu} \partial_{\nu} f(y)
$$

In the following, we show that the matrix $g_{\mu \nu}(y)$ is a metric on $\mathcal{M}$.

Let us consider the expansion (2.15) in which $|\epsilon|=\sqrt{\delta_{\mu \nu} \epsilon^{\mu} \epsilon^{\nu}}$ is much smaller than the typical scale of $\mathcal{M}$. If both $y$ and $y+\epsilon$ are contained in $\mathcal{M}$, we can regard $\epsilon$ as a tangent vector on $\mathcal{M}$, and we denote $\epsilon=\epsilon_{\|}$. In this case, since $f(y)=f\left(y+\epsilon_{\|}\right)=0$, we have

$$
g_{\mu \nu}(y) \epsilon_{\|}^{\mu} \epsilon_{\|}^{\nu}=\left|\epsilon_{\|}\right|^{2} .
$$

On the other hand, let us consider the case in which $\epsilon=\epsilon_{\perp}$ is a normal vector at $y \in \mathcal{M}$. In this case, as shown in Appendix $\underline{\mathrm{C}}, f\left(y+\epsilon_{\perp}\right)$ is given by the distance between $y$ and $y+\epsilon_{\perp}$ as

$$
f\left(y+\epsilon_{\perp}\right)=\frac{1}{2}\left|\epsilon_{\perp}\right|^{2} .
$$

By comparing this to Eq. (2.15) with $\epsilon=\epsilon_{\perp}$, we obtain

$$
g_{\mu \nu}(y) \epsilon_{\perp}^{\mu} \epsilon_{\perp}^{\nu}=0
$$

Equations (3.3) and (3.5) show that $g_{\mu \nu}(y)$ is a projection to the tangent space $T \mathcal{M}_{y}$ at $y \in \mathcal{M}$. Thus, it satisfies

$$
g_{\mu \nu}(y) g_{\rho}^{\nu}(y)=g_{\mu \rho}(y) \quad(y \in \mathcal{M})
$$

Here, we again emphasize that the index $\nu$ is raised using the Kronecker delta, so that $g_{\rho}^{\nu}=g_{\nu \rho}$. Since this implies that $g_{\mu \nu}(y)$ is positive and nondegenerate on $\mathcal{M}$, it gives a metric on $\mathcal{M}$. The line element on $\mathcal{M}$ can be written as

$$
d s^{2}=g_{\mu \nu}(y) d y^{\mu} d y^{\nu}
$$

It is interesting that the positivity of the metric comes from the negativity of the secondorder correction to the ground state energy in the perturbation theory of quantum mechanics.

Note that, for any $y \in \mathcal{M}$, the dimension of $\mathcal{M}$ is given by the trace of $g_{\mu \nu}(y)^{7}$,

$$
\operatorname{dim} \mathcal{M}=\sum_{\mu=1}^{D} g_{\mu \mu}(y)
$$

\footnotetext{
${ }^{7}$ If $\mathcal{M}$ consists of some disconnected components, the trace of $g_{\mu \nu}(y)$ gives the dimension of the component which contains $y$.
} 
This expression and (3.1) relate the dimension of $\mathcal{M}$ with the matrix configurations.

\subsection{Levi-Civita connection}

Let $A^{\mu}(y)$ and $B^{\mu}(y)$ be tangent vector fields which satisfy $g^{\mu}{ }_{\nu}(y) A^{\nu}(y)=A^{\mu}(y)$ and $g^{\mu}{ }_{\nu}(y) B^{\nu}(y)=B^{\mu}(y)$ for $y \in \mathcal{M}$. We define the covariant derivative on $\mathcal{M}$ by

$$
\left(\nabla_{B} A\right)^{\mu}=B^{\nu}\left(\partial_{\nu} A^{\mu}+\Gamma_{\nu \rho}^{\mu} A^{\rho}\right)
$$

The connection $\Gamma_{\nu \rho}^{\mu}$ is chosen such that the image of the covariant derivative (3.9) is again a tangent vector, namely,

$$
g^{\mu}{ }_{\nu}\left(\nabla_{B} A\right)^{\nu}=\left(\nabla_{B} A\right)^{\mu} .
$$

By using (3.2) and

$$
B^{\rho}(y)\left\{\partial_{\rho} g_{\mu \sigma}(y)-g_{\mu \nu}(y) \partial_{\rho} g_{\sigma}^{\nu}(y)-g_{\nu \sigma}(y) \partial_{\rho} g^{\nu}{ }_{\mu}(y)\right\}=0 \quad(y \in \mathcal{M}),
$$

which is obtained by differentiating (3.6), we find a simple solution to (3.10) as

$$
\Gamma_{\nu \rho}^{\mu}=\left(\partial^{\sigma} \partial^{\mu} f\right)\left(\partial_{\sigma} \partial_{\nu} \partial_{\rho} f\right) .
$$

We also find that $\left(\nabla_{B} g\right)_{\mu \nu}=0$. This means that (3.12) is the Levi-Civita connection associated with the metric $g_{\mu \nu}$. The expression (3.12) together with (2.14) relates the connection and the matrix elements of $X^{\mu}$.

\subsection{Curvature}

Let $A, B, C$ be tangent vector fields on $\mathcal{M}$. The curvature tensor is defined by

$$
R(A, B) C=\left[\nabla_{A}, \nabla_{B}\right] C-\nabla_{[A, B]} C,
$$

where $[A, B]$ represents the Lie bracket of the vector fields,

$$
[A, B]^{\mu}=A^{\nu} \partial_{\nu} B^{\mu}-B^{\nu} \partial_{\nu} A^{\mu}
$$

Note that this is also a tangent vector satisfying $g_{\mu \nu}[A, B]^{\nu}=[A, B]^{\mu}$. In terms of components, we can write the curvature as

$$
(R(A, B) C)^{\mu}=A^{\nu} B^{\rho} C^{\sigma} R^{\mu}{ }_{\sigma \nu \rho}
$$




$$
=A^{\nu} B^{\rho} C^{\sigma}\left\{\left(\partial^{\mu} \partial_{\nu} \partial_{\lambda} f\right)\left(\partial_{\rho} \partial_{\sigma} \partial^{\lambda} f\right)-\left(\partial^{\mu} \partial_{\rho} \partial_{\lambda} f\right)\left(\partial_{\nu} \partial_{\sigma} \partial^{\lambda} f\right)\right\} .
$$

By contracting the indices of $R_{\sigma \nu \rho}^{\mu}(y)$ using $g_{\mu \nu}(y)$, we can also write the Ricci tensor or the Ricci scalar in terms of the derivatives of $f(y)$. These expressions and (2.14) give relations between the curvature tensors and the matrices.

\subsection{Poisson tensor}

We consider a real antisymmetric $D \times D$ matrix on $\mathcal{M}$ defined by

$$
W^{\mu \nu}(y)=\lim _{N \rightarrow \infty} c\left\langle 0, y\left|\left[X^{\mu}, X^{\nu}\right]\right| 0, y\right\rangle,
$$

where $c$ is a pure imaginary $N$-dependent normalization constant. The constant $c$ is chosen so that $c\left\langle 0, y\left|\left[X^{\mu}, X^{\nu}\right]\right| 0, y\right\rangle$ becomes $\mathcal{O}\left(N^{0}\right)$ in the large- $N$ limit (see the next section for concrete examples). $W^{\mu \nu}(y)$ is a tangent bivector on $\mathcal{M}$ and satisfies the Jacobi identity as we will show below. This means that $W^{\mu \nu}(y)$ is a Poisson tensor on $\mathcal{M}$.

We first show that $W^{\mu \nu}(y)$ is a tangent bivector, namely, it satisfies $g_{\mu \rho}(y) W^{\rho \nu}(y)=$ $W^{\mu \nu}(y)$ for $y \in \mathcal{M}$. In order to prove this, the following relation is useful:

$$
c\left\langle 0, y\left|\left[X^{\mu}, X^{\nu}\right]\right| 0, y\right\rangle\left\langle 0, y\left|X_{\nu}\right| n, y\right\rangle \sim c\left(E_{n}(y)-E_{0}(y)\right)\left\langle 0, y\left|X^{\mu}\right| n, y\right\rangle,
$$

where $\sim$ stands for an equality for the leading-order terms in the large- $N$ limit. This can be shown as follows. By using (2.19) and (2.20), we obtain

$$
c\left\langle 0, y\left|\left[X^{\mu}, X^{\nu}\right]\right| 0, y\right\rangle\left\langle 0, y\left|X_{\nu}\right| n, y\right\rangle \sim \frac{c}{2}\left\langle 0, y\left|\left\{X^{\nu}-y^{\nu},\left[X^{\mu}, X_{\nu}-y_{\nu}\right]\right\}\right| n, y\right\rangle .
$$

Then noticing that $\left\{X^{\nu}-y^{\nu},\left[X^{\mu}, X_{\nu}-y_{\nu}\right]\right\}=2\left[X^{\mu}, H(y)\right]$, we obtain (3.17). Now, let us calculate

$$
g^{\mu}{ }_{\rho}(y) W^{\rho \nu}(y)=\lim _{N \rightarrow \infty}\left[2 \operatorname{Re}\left\{c \sum_{n \neq 0} \frac{\left\langle 0, y\left|X^{\mu}\right| n, y\right\rangle\left\langle n, y\left|X^{\rho}\right| 0, y\right\rangle}{E_{n}(y)-E_{0}(y)}\left\langle 0, y\left|\left[X_{\rho}, X^{\nu}\right]\right| 0, y\right\rangle\right\}\right] .
$$

By using (3.17) and the completeness relation $\sum_{n=0}^{N-1}|n, y\rangle\langle n, y|=1$, we obtain

$$
g_{\rho}^{\mu}(y) W^{\rho \nu}(y)=\lim _{N \rightarrow \infty}\left[2 \operatorname{Re}\left\{c\left\langle 0, y\left|X^{\mu} X^{\nu}\right| 0, y\right\rangle-c\left\langle 0, y\left|X^{\mu}\right| 0, y\right\rangle\left\langle 0, y\left|X^{\nu}\right| 0, y\right\rangle\right\}\right] .
$$

Since we have assumed that $c$ is pure imaginary and $X^{\mu}$ are Hermitian, the second term is zero, while the first term is equal to $W^{\mu \nu}(y)$. Thus, we have shown that $W^{\mu \nu}(y)$ is a tangent bivector on $\mathcal{M}$. The Jacobi identity of the Poisson bracket can be shown in a similar way. We leave the proof to Appendix D. 


\section{Examples}

In this section, we consider some examples.

\subsection{Fuzzy sphere}

Our first example is the fuzzy sphere, which is given by

$$
X_{\mu}=\frac{2}{\sqrt{N^{2}-1}} L_{\mu}(\mu=1,2,3),
$$

where $L_{\mu}$ are the $N$-dimensional representation matrices of the $S O(3)$ generators satisfying $\left[L_{\mu}, L_{\nu}\right]=i \epsilon_{\mu \nu \rho} L_{\rho}$ and $\sum_{\mu=1}^{3} L_{\mu}^{2}=\frac{N^{2}-1}{4} \mathbf{1}_{N \times N}$. The normalization in (4.1) is chosen in such a way that the radius of the sphere becomes one: $X^{\mu} X_{\mu}=\mathbf{1}_{N \times N}$. The corresponding Hamiltonian (2.5) is given by

$$
H(y)=\frac{1+|y|^{2}}{2}-\frac{2 L_{\mu} y^{\mu}}{\sqrt{N^{2}-1}} .
$$

Here, we notice that for any $S O(3)$ rotation, $L_{\mu} \rightarrow \Lambda_{\mu}{ }^{\nu} L_{\nu}$, there exists a unitary transformation which reproduces this rotation:

$$
R L_{\mu} R^{\dagger}=\Lambda_{\mu}{ }^{\nu} L_{\nu}
$$

See Appendix B for this transformation. Hence it suffices to consider the case where $y=\left(y^{1}, y^{2}, y^{3}\right)=(0,0, r)$ with $r=|y| \geq 0$. Then, the Hamiltonian reduces to

$$
H(y)=\frac{1+|y|^{2}}{2}-\frac{2 L_{3}|y|}{\sqrt{N^{2}-1}} .
$$

The ground state of this Hamiltonian is given by the highest eigenstate of $L_{3}$ with the eigenvalue $(N-1) / 2$. Thus, we find that the function $f(y)$ is given by

$$
f(y)=\frac{1}{2}(1-|y|)^{2}
$$

Therefore, the classical space $\mathcal{M}$, which is given as a set of zeros of $f(y)$, is indeed a sphere with the unit radius. Here, we remark that $E_{0}(y)$ is a smooth function for any point $y$ apart from the origin, so that the smoothness condition assumed in the previous section is satisfied. 
We can also compute the metric either from (3.1) or (3.2). The result is given by

$$
g_{\mu \nu}(y)=\frac{1}{|y|}\left(\delta_{\mu \nu}-\frac{y_{\mu} y_{\nu}}{|y|^{2}}\right) .
$$

For $y \in \mathcal{M}$, this is indeed a projection to the tangent space and (3.7) gives the standard line element on $S^{2}$. It is an easy exercise to compute the curvature tensors from the

definition in Sec. 3.3 and check that the Ricci scalar is equal to 2 and agrees with the known value for the unit sphere.

The Poisson tensor (3.16) is given by

$$
W^{\mu \nu}(y)=i \epsilon^{\mu \nu \rho} \lim _{N \rightarrow \infty} \frac{2 c}{\sqrt{N^{2}-1}}\left\langle 0, y\left|X_{\rho}\right| 0, y\right\rangle .
$$

If we put $c=\frac{-i}{2} \sqrt{N^{2}-1}$, we obtain

$$
W^{\mu \nu}(y)=\epsilon^{\mu \nu \rho} y_{\rho}
$$

where we have used (2.17). This is the standard Poisson tensor on $S^{2}$ embedded in $\mathbf{R}^{3}$.

\subsection{Fuzzy torus}

The fuzzy torus is defined in terms of the algebra of two unitary matrices $U$ and $V$,

$$
V U=e^{i \theta} U V
$$

where $\theta=2 \pi / N$. The matrices $U$ and $V$ can be represented by the clock and shift matrices as

$$
\begin{aligned}
& U_{m n}=\delta_{m n} e^{i n \theta}, \\
& V_{m n}=\delta_{m+1 n},
\end{aligned}
$$

where $m, n=0,1, \cdots, N-1$ and $\delta_{m n}$ is the cyclic Kronecker delta which satisfies $\delta_{N n} \equiv$ $\delta_{0 n}$. By introducing four Hermitian matrices $X^{\mu}(\mu=1,2,3,4)$ as the real and imaginary parts of $U$ and $V$ as

$$
U=X^{1}+i X^{2}, \quad V=X^{3}+i X^{4}
$$

we can regard the fuzzy torus as the noncommutative Clifford torus embedded in $\mathbf{R}^{4}$. Note that $X^{1}$ and $X^{2}$ commute with each other and satisfy $\left(X^{1}\right)^{2}+\left(X^{2}\right)^{2}=\mathbf{1}_{N \times N}$ because of 
the unitarity condition of $U$, and so do $X^{3}$ and $X^{4}$ because of the unitarity condition of $V$. The Hamiltonian (2.5) is given by

$$
H(y)=\frac{1}{2}(U-z)\left(U^{\dagger}-\bar{z}\right)+\frac{1}{2}(V-w)\left(V^{\dagger}-\bar{w}\right)
$$

where $z=y^{1}+i y^{2}$ and $w=y^{3}+i y^{4}$. As shown in Appendix $\mathrm{E}$, in this case the function $f(y)$ is given by

$$
f(y)=\frac{1}{2}(1-|z|)^{2}+\frac{1}{2}(1-|w|)^{2} .
$$

Hence, the classical space $\mathcal{M}$ is indeed the Clifford torus given by $|z|=|w|=1$.

We can also obtain the metric from (3.2) as

$$
g_{a b}(y)=\frac{1}{|z|}\left(\delta_{a b}-\frac{y_{a} y_{b}}{|z|^{2}}\right), \quad g_{a \alpha}(y)=0, \quad g_{\alpha \beta}(y)=\frac{1}{|w|}\left(\delta_{\alpha \beta}-\frac{y_{\alpha} y_{\beta}}{|w|^{2}}\right),
$$

where $a, b=1,2$ and $\alpha, \beta=3,4$. We can see that $g_{\mu \nu}$ is a projection operator for $y \in \mathcal{M}$ and gives the standard metric of the Clifford torus.

In order to compute the Poisson tensor, we rewrite the algebra (4.9) using $X^{\mu}$ as

$$
\begin{aligned}
& {\left[X_{1} \pm i X_{2}, X_{3} \pm i X_{4}\right] \sim i \theta\left(X_{1} \pm i X_{2}\right)\left(X_{3} \pm i X_{4}\right)} \\
& {\left[X_{1} \pm i X_{2}, X_{3} \mp i X_{4}\right] \sim-i \theta\left(X_{1} \pm i X_{2}\right)\left(X_{3} \mp i X_{4}\right)}
\end{aligned}
$$

where we have neglected higher-order terms in $\theta$. By putting $c=i / \theta$ in (3.16), we obtain the Poisson tensor on the Clifford torus:

$$
W^{\mu \nu}(y)=\left(\begin{array}{cccc}
0 & 0 & y^{2} y^{4} & -y^{2} y^{3} \\
0 & 0 & -y^{1} y^{4} & y^{1} y^{3} \\
-y^{2} y^{4} & y^{1} y^{4} & 0 & 0 \\
y^{2} y^{3} & -y^{1} y^{3} & 0 & 0
\end{array}\right) .
$$

\section{Infinite-dimensional matrices}

As far as the Hamiltonian (2.5) has a discrete spectrum, it will be possible to apply the same formulation to matrices with an infinite size. In this case, we assume that we are given a one-parameter family of $D$ operators $X_{\mu}^{(\hbar)}$ acting on an infinite-dimensional Hilbert space $\mathcal{H}_{\hbar}$, where $\hbar$ is a non-negative parameter. We replace the large- $N$ limit considered in the previous sections with the limit of $\hbar \rightarrow 0$ and the finite sums such as $\sum_{n=0}^{N-1}$ with 
the infinite sums $\sum_{n=0}^{\infty}$ appropriately. In this section, by considering a generic example, we will demonstrate that our formulation also works for infinite-dimensional matrices.

In this section, we work with the notation as follows. We decompose the indices $\mu, \nu, \rho$, which run from 1 to $D$, to two indices as $\mu \rightarrow\{A, I\}$. The indices $A, B, C, \cdots$ run from 1 to 4 , while $I, J, K, \cdots$ run from 5 to $D$. We further decompose $A \rightarrow\{a, \alpha\}$, so that $a, b, \cdots$ run from 1 to 2 and $\alpha, \beta, \cdots$ run from 3 to 4 .

We consider a four dimensional noncommutative plane with some fluctuations embedded in $\mathbf{R}^{D}$, which is given by

$$
\begin{aligned}
& X^{A}=\hat{q}^{A}+\tilde{X}^{A} \quad(A=1,2,3,4), \\
& X^{I}=\tilde{X}^{I} \quad(I=5,6, \cdots, D) .
\end{aligned}
$$

Here $\hat{q}^{A}$ are the operators satisfying

$$
\left[\hat{q}^{a}, \hat{q}^{b}\right]=i \epsilon^{a b} \theta, \quad\left[\hat{q}^{\alpha}, \hat{q}^{\beta}\right]=i \epsilon^{\alpha \beta} \theta^{\prime}, \quad\left[\hat{q}^{a}, \hat{q}^{\alpha}\right]=0
$$

where $\epsilon^{a b}$ and $\epsilon^{\alpha \beta}$ are $2 \times 2$ antisymmetric matrices with $\epsilon^{12}=1$ and $\epsilon^{34}=1 . \theta$ and $\theta^{\prime}$ are the noncommutative parameters which are assumed to vanish in the classical limit $\hbar \rightarrow 0$. We assume that the fluctuations $\left\{\tilde{X}^{\mu}\right\}$ are of the form

$$
\tilde{X}^{\mu}=\int \frac{d^{4} k}{(2 \pi)^{4}} \int d^{4} x e^{-i k \cdot x} e^{i k \cdot \hat{q}} \phi^{\mu}(x) \quad(\mu=1,2, \cdots, D),
$$

where $k \cdot x=\sum_{A=1}^{4} k_{A} x^{A}$ is the four-dimensional inner product and the Weyl symbols $\phi^{\mu}(x)$ are real functions on $\mathbf{R}^{4}$. Here, we emphasize that $\phi^{\mu}(x)$ are just C-number valued functions and they depend only on the four-dimensional coordinates $x_{A}$. We assume that $\left|\phi^{\mu}(x)\right|$ are small, so that we can treat $\tilde{X}^{\mu}$ perturbatively. The configuration (5.1) is often considered in the context of the description of gauge theories on the noncommutative plane in terms of matrix models [16].

For the configuration (5.1), the Hamiltonian (2.5) is given by

$$
H(y)=H_{0}(y)+H_{1}(y)+H_{2}(y)
$$

where $H_{0}(y), H_{1}(y)$ and $H_{2}(y)$ are defined by

$$
H_{0}(y)=\frac{1}{2}\left(\hat{q}_{A}-y_{A}\right)^{2}+\frac{1}{2} y_{I}^{2}, \quad H_{1}(y)=\frac{1}{2}\left\{\hat{q}_{A}-y_{A}, \tilde{X}^{A}\right\}-\tilde{X}_{I} y^{I}, \quad H_{2}(y)=\frac{1}{2} \tilde{X}_{\mu}^{2} .
$$


The subscripts of the Hamiltonians indicate the degrees of the perturbation. The zerothorder Hamiltonian $H_{0}(y)$ is just the two-dimensional harmonic oscillator. We introduce eigenstates of $H_{0}(y)$ by

$$
\begin{aligned}
& H_{0}(y)|m, n, y\rangle=E_{m, n}^{(0)}|m, n, y\rangle, \\
& E_{m, n}^{(0)}=\theta\left(m+\frac{1}{2}\right)+\theta^{\prime}\left(n+\frac{1}{2}\right)+\frac{1}{2} y_{I}^{2} .
\end{aligned}
$$

See Appendix $\mathrm{F}$ for explicit forms of the wave functions. In the following, in order to avoid the degeneration of $H_{0}(y)$, we assume that $\theta$ and $\theta^{\prime}$ are written as $\theta=a \hbar$ and $\theta^{\prime}=b \hbar$, where $a$ and $b$ are rational and irrational constants, respectively. Under this assumption, we can naively apply the formulas in the previous sections.

In Appendix $\mathrm{F}$, we compute the ground state energy of the full Hamiltonian $H(y)$ up to the second-order perturbation in $\tilde{X}_{\mu}$ and derive the following form of $f(y)$ :

$$
f(y)=\frac{1}{2}\left\{y^{I}-\phi^{I}(y)+\phi^{A}(y) \partial_{A} \phi^{I}(y)\right\} h_{I J}(y)\left\{y^{J}-\phi^{J}(y)+\phi^{B}(y) \partial_{B} \phi^{J}(y)\right\}+\mathcal{O}\left(\phi^{3}\right),
$$

where

$$
h_{I J}(y)=\delta_{I J}-\partial^{A} \phi_{I}(y) \partial_{A} \phi_{J}(y)
$$

Hence, the classical space $\mathcal{M}$ is given by a four-dimensional surface in $\mathbf{R}^{D}$ defined by

$$
y_{I}=\phi_{I}(y)-\phi^{A}(y) \partial_{A} \phi_{I}(y)+\mathcal{O}\left(\phi^{3}\right) \quad(I=5,6, \cdots, D) .
$$

When $\phi^{\mu}=0$, the above equation reduces to $y_{I}=0$ and $\mathcal{M}$ is just the four-dimensional flat space parametrized by $\left\{y_{A}\right\}$. Equation (5.9) shows that $\mathcal{M}$ also fluctuates along the transverse directions when $\phi^{\mu}$ are turned on.

From Eq. (3.1) or (3.2), we can read off the metric on $\mathcal{M}$ as

$$
\begin{aligned}
& g_{A B}(y)=\delta_{A B}-\partial_{A} \phi_{I}(y) \partial_{B} \phi^{I}(y)+\mathcal{O}\left(\phi^{3}\right), \\
& g_{A I}(y)=\partial_{A} \phi_{I}(y)-\partial_{A}\left(\phi^{B}(y) \partial_{B} \phi_{I}(y)\right)+\mathcal{O}\left(\phi^{3}\right), \\
& g_{I J}(y)=\partial^{A} \phi_{I}(y) \partial_{A} \phi_{J}(y)+\mathcal{O}\left(\phi^{3}\right) .
\end{aligned}
$$

It is easy to check the projection property (3.6) for this metric. By substituting the metric (5.10) into (3.7), we obtain the line element

$$
d s^{2}=\left(\delta_{A B}+\partial_{A} \phi^{I}(y) \partial_{B} \phi_{I}(y)\right) d y^{A} d y^{B}+\mathcal{O}\left(\phi^{3}\right),
$$


where we have used $d y^{I}=g_{I A}(y) d y^{A}$ obtained from (5.9). By introducing new coordinates $\sigma^{A}$ by $y^{A}=\sigma^{A}+\phi^{A}(\sigma)$, we can rewrite the line element as

$$
d s^{2}=\left[\left(\delta_{A}^{C}+\partial_{A} \phi^{C}(\sigma)\right)\left(\delta_{B C}+\partial_{B} \phi_{C}(\sigma)\right)+\partial_{A} \phi_{I}(\sigma) \partial_{B} \phi^{I}(\sigma)\right] d \sigma^{A} d \sigma^{B}+\mathcal{O}\left(\phi^{3}\right) .
$$

This is nothing but the induced metric associated with the embedding function given by $X^{A}(\sigma)=\sigma^{A}+\phi^{A}(\sigma)$ and $X^{I}(\sigma)=\phi^{I}(\sigma)$.

Through a similar calculation, we can obtain the following form for the Poisson tensor,

$$
\begin{aligned}
W^{A B}(y)= & B^{A B}+B^{A C} \partial_{C} \phi^{B}(y)-B^{B C} \partial_{C} \phi^{A}(y)+B^{C D} \partial_{C} \phi^{A}(y) \partial_{D} \phi^{B}(y) \\
& -B^{A D} \phi^{C}(y) \partial_{C} \partial_{D} \phi^{B}(y)+B^{B D} \phi^{C}(y) \partial_{C} \partial_{D} \phi^{A}(y)+\mathcal{O}\left(\phi^{3}\right) \\
W^{A I}(y)= & B^{A B} \partial_{B} \phi^{I}(y)+B^{B C} \partial_{B} \phi^{A}(y) \partial_{C} \phi^{I}(y)-B^{A D} \phi^{C}(y) \partial_{C} \partial_{D} \phi^{I}(y)+\mathcal{O}\left(\phi^{3}\right) \\
W^{I J}(y)= & B^{A B} \partial_{A} \phi^{I}(y) \partial_{B} \phi^{J}(y)+\mathcal{O}\left(\phi^{3}\right) .
\end{aligned}
$$

Here, we have defined a real antisymmetric matrix $B^{A B}$ by $\left[\hat{q}^{A}, \hat{q}^{B}\right]=i \hbar B^{A B}$ and we have chosen the constant $c$ in (13.16) as $c=-i / \hbar$. From (5.10) and (5.13), one can easily check that $W^{\mu \nu}(y)$ is a tangent bivector and its Poisson bracket satisfies the Jacobi identity.

\section{Summary and discussion}

In this paper, we proposed a novel method of finding the classical limit of matrix geometries. We first introduced the notion of coherent states for general matrix geometry described by a large- $N$ sequence of $D$ Hermitian matrices $\left(X^{1}, X^{2}, \cdots, X^{D}\right)$. We then defined the classical space $\mathcal{M}$ as a set of all coherent states. Assuming that the set of coherent states forms a smooth manifold and so does $\mathcal{M}$, we also found expressions for various geometric objects on $\mathcal{M}$ (metric, connection, curvature and Poisson tensor) in terms of the $D$ matrices.

The usage of our result on the geometric objects is limited to the cases where the classical space is a smooth manifold. Probably, if we consider a general matrix sequence, the corresponding classical space is most likely to be an empty set. In order to have a nontrivial space, there must be a subspace of the Hilbert space on which the $D$ matrices become mutually commuting with each other. Though this is not the case for a general sequence, there are physically relevant situations where such commuting matrices 
naturally arise. In matrix models of the Yang-Mills type (i.e. models with commutator interactions), typical values of the commutators might become very small in the strong coupling region, since one can always take a normalization such that the coupling constant appears only in front of the commutators. As stated in [17], this gives a reasonable mechanism for the emergence of space in matrix models. Thus, in the strong coupling regime of matrix models, we can expect that nontrivial manifolds emerge and they can be visualized by using our method.

Even if the space is nonempty, we needed to assume the smoothness condition to define the geometric objects. This condition is necessary to avoid some singular cases. The simplest singular example can be found for some special cases of diagonal matrices. Suppose that all of the $D$ matrices are diagonal for any $N$. In this case, $\mathcal{M}$ is just given by a discrete set of points corresponding to the positions of the eigenvalues in $\mathbf{R}^{D}$. Let us consider a further special case where the eigenvalue distribution becomes dense and continuous in a certain fixed region of $\mathbf{R}^{D}$ in the large- $N$ limit, as the limit considered in the context of the large- $N$ reduction [18 21]. In this limit, $\mathcal{M}$ becomes continuous manifold given by the eigenvalue distribution in the large- $N$ limit. However, near the large- $N$ limit, the ground state energy $E_{0}(y)$ is nondifferentiable almost everywhere on the dense region. Hence the formulation in Sec. 3 does not work. This example suggests that the off-diagonal elements are essential to describe the metric and the other geometric objects. Probably this kind of singularity should also be related with the topology change of membranes in $\mathrm{M}$ theory and we hope to find clear criteria to distinguish such singular configurations of matrices.

Let us make some comments on our results. Firstly, our result can easily be applied to small perturbations around smooth configurations. For finite $N$ matrices, let us consider an expansion of the matrices, $X^{\mu}=\hat{X}^{\mu}+h^{\mu}(\hat{X})$. Here, $\hat{X}^{\mu}$ are (a large- $N$ sequence of) matrices which satisfy the smoothness condition and have an associated classical manifold M. $h^{\mu}(\hat{X})$ are smooth polynomial functions which represent small fluctuations. In this case, by treating $h^{\mu}(\hat{X})$ as perturbation, from (2.18) $-(2.20)$, one can show that the classical space for $X^{\mu}$ is given by $\left\{y \in \mathbf{R}^{D} \mid y^{\mu}=\hat{y}^{\mu}+\left(\delta_{\nu}^{\mu}-\hat{g}^{\mu}{ }_{\nu}(\hat{y})\right) h^{\nu}(\hat{y}), \quad \hat{y} \in \mathcal{M}\right\}$, where $\hat{g}_{\mu \nu}$ is the metric of $\mathcal{M}$. Thus, the perturbed geometry is again a smooth manifold.

Secondly, if the matrices are thought of as the transverse coordinates of D-branes, the 
classical space just corresponds to the classical shape of the D-branes. For example, the fuzzy sphere formed by matrices of D0-branes can be interpreted as the D2-brane arising via the Myers effect [22]. In this phenomena, the net D2-brane charge is zero, while there is a nontrivial gauge flux on D2-brane, which induces a coupling to the R-R 1-form. This gauge field will be realized as the Berry connection with respect to the Hamiltonian (2.5) in our formulation. For example in the fuzzy sphere case, let us define $A_{\mu}=-i\left\langle\Omega\left|\partial_{\mu}\right| \Omega\right\rangle$, where $|\Omega\rangle$ is the Bloch coherent state defined in $(\underline{B .3})$ and $\mu=\theta, \phi$. Then we can find that the gauge field coincides with the Dirac monopole configuration, which agrees with the gauge field induced on D2-branes [22]. Although we have discussed local properties of the coherent states in this paper, it will also be interesting to study global aspects in connection with the Berry phase of the Hamiltonian (2.5).

Now, let us discuss some possible applications of our result. Since all the geometric objects defined in this paper are invariant under the $U(N)$ gauge transformation $X^{\mu} \rightarrow$ $U X^{\mu} U^{\dagger}$, they can be regarded as observables in matrix models. These new observables will be useful in understanding geometric aspects of matrix models. For example, they can be used to figure out the classical shape of D-branes in matrix models [12] and also to study the emergence of space-time in matrix models [23, 24].

Our formulation also works for higher-dimensional gauge theories, and we can define the similar observables in gauge theories, which are useful in studying the gauge/gravity correspondence [25]. In the gauge/gravity correspondence, the background geometry of the string theory is expected to be emergent in the strong coupling limit of the corresponding gauge theory [26 28]. Though field configurations on the gauge theory side are considered to encode the information of the background geometry, a general prescription to see this is not known yet. Our observables will offer a new method of translating field configurations to the corresponding background geometry.

Those observables will also be quite useful in computer simulations of matrix models [24,29,30]. By using a computer, one can easily compute the observables from given matrix configurations and see the geometric properties. Furthermore, although we considered the strict large- $N$ limit when we defined the geometric objects, our formulation also works at finite $N$ as an approximation, which is rather easily accessible from numerical simulations 8 .

\footnotetext{
${ }^{8}$ For example, the dimension of the fuzzy sphere (4.1) with $N=50$ can be read off from the value of $g_{\mu \mu}$ at the minimum of the ground state energy. One can find $g_{\mu \mu}=2.04$, which gives a very good
} 
Although we only considered the problem of the classical limit in this paper, finding a general construction method of the matrix regularization is also a very important problem. In our formulation, coherent states introduce the notion of locality for matrix configurations. This idea may help to construct the matrix regularization for a general manifold. For instance, for a given manifold, one can first discretize the manifold to a fine lattice, and let the coordinates of each lattice cite to be diagonal elements of the matrices. In order to determine the off-diagonal elements, one may refer to the representation of the coordinate operators on the noncommutative plane in the basis of the coherent states. If any matrix geometry looks locally like the noncommutative plane, the off-diagonal elements should be locally the same as those on the noncommutative plane. If so, by gluing those components along the tangent directions, it will be possible to construct the $D$ Hermitian matrices which provide the matrix regularization.

We pursue these directions and hope to report these issues in the near future.

\section{Acknowledgements}

This work was supported, in part, by Program to Disseminate Tenure Tracking System, MEXT, Japan.

\section{A Canonical coherent states}

In this Appendix, we consider the canonical coherent states and summarize their properties [31]. We start with the algebra of the creation-annihilation operators,

$$
\left[\hat{a}, \hat{a}^{\dagger}\right]=1 .
$$

The vacuum state is defined by

$$
\hat{a}|0\rangle=0,
$$

as well as $\langle 0 \mid 0\rangle=1$. We consider the Hilbert space given by

$$
\mathcal{H}=\operatorname{span}\left\{\left(\hat{a}^{\dagger}\right)^{n}|0\rangle \mid n \in \mathbf{Z}, n \geq 0\right\} .
$$

approximation of $\operatorname{dim} S^{2}=2$. This kind of analysis will be possible for more general configurations. 
The canonical coherent states are elements of $\mathcal{H}$ parametrized by a complex number $z \in \mathbf{C}$, and defined as

$$
|z\rangle=e^{z \hat{a}^{\dagger}-\bar{z} \hat{a}}|0\rangle
$$

Let us introduce the Hamiltonian of the harmonic oscillator,

$$
H=\hbar\left(\hat{a}^{\dagger} \hat{a}+\frac{1}{2}\right)
$$

and its eigenstates

$$
H|n\rangle=E_{n}|n\rangle, \quad E_{n}=\hbar\left(n+\frac{1}{2}\right), \quad|n\rangle=\frac{1}{\sqrt{n !}}\left(\hat{a}^{\dagger}\right)^{n}|0\rangle \quad(n=1,2, \cdots) .
$$

The coherent states can be written in terms of $|n\rangle$ as

$$
|z\rangle=e^{-\frac{1}{2}|z|^{2}} \sum_{n=0}^{\infty} \frac{z^{n}}{\sqrt{n !}}|n\rangle .
$$

The canonical coherent states have the following properties:

(i) $\left\langle z_{1} \mid z_{2}\right\rangle=e^{-\frac{\left|z_{1}\right|^{2}}{2}-\frac{\left|z_{2}\right|^{2}}{2}+\bar{z}_{1} z_{2}}$

(ii) $\int \frac{d^{2} z}{\pi}|z\rangle\langle z|=1 \quad$ (resolution of identity),

(iii) $\hat{a}|z\rangle=z|z\rangle$,

(iv) $|z\rangle$ is the ground state of the shifted Hamiltonian,

$$
H(z)=\hbar\left(\left(\hat{a}^{\dagger}-\bar{z}\right)(\hat{a}-z)+\frac{1}{2}\right),
$$

(v) $|z\rangle$ saturates the uncertainty inequality.

The first and the second properties can be derived from (A.7) and the completeness relation $\sum_{n}|n\rangle\langle n|=1$. The integration measure in (B.7) is defined as the flat measure for the real and imaginary parts of $z, \int d^{2} z:=\int_{-\infty}^{\infty} d(\operatorname{Re} z) \int_{-\infty}^{\infty} d(\operatorname{Im} z)$. The third, fourth and fifth properties follow from the fact that the unitary operator $U(z)=e^{z \hat{a}^{\dagger}-\bar{z} \hat{a}}$, which appears in Eq. (A.4), is the translation operator $U^{\dagger}(z) \hat{a} U(z)=\hat{a}+z$. 


\section{B Bloch coherent states}

Coherent states for the fuzzy sphere are called the Bloch (spin) coherent states [31] 9 . The fuzzy sphere is described by the $S U(2)$ Lie algebra:

$$
\left[L_{i}, L_{j}\right]=i \epsilon_{i j k} L_{k}
$$

We consider the spin $J$ representation, so that the $L_{i}$ are $(2 J+1) \times(2 J+1)$ matrices. We introduce the standard basis for this representation space, which satisfy

$$
\begin{aligned}
& L_{3}|J m\rangle=m|J m\rangle, \\
& L_{ \pm}|J m\rangle=\sqrt{(J \mp m)(J \pm m+1)}|J m \pm 1\rangle,
\end{aligned}
$$

where $L_{ \pm}=L_{1} \pm i L_{2}, m=-J,-J+1, \cdots, J$ and the states are normalized as $\left\langle J m \mid J m^{\prime}\right\rangle=$ $\delta_{m m^{\prime}}$. The Bloch coherent states are parametrized by the coordinates on $S^{2}, \Omega=(\theta, \phi)$, where $0 \leq \theta \leq \pi$ and $0<\phi \leq 2 \pi$, and defined by

$$
|\Omega\rangle=e^{\frac{1}{2} \theta e^{i \phi} L_{-}-\frac{1}{2} \theta e^{-i \phi} L_{+}}|J J\rangle .
$$

By using the Baker-Canbell-Hausdorff formula, they can also be written as

$$
|\Omega\rangle=e^{z L_{-}} e^{-L_{3} \log \left(1+|z|^{2}\right)} e^{-\bar{z} L_{+}}|J J\rangle,
$$

where $z=\tan \frac{\theta}{2} e^{i \phi}$. By acting the operators onto $|J J\rangle$, one can rewrite them as

$$
\begin{aligned}
|\Omega\rangle & =\frac{1}{\left(1+|z|^{2}\right)^{J}} \sum_{m=-J}^{J} z^{J-m}\left(\begin{array}{c}
2 J \\
J+m
\end{array}\right)^{\frac{1}{2}}|J m\rangle \\
& =\sum_{m=-J}^{J}\left(\begin{array}{c}
2 J \\
J+m
\end{array}\right)^{\frac{1}{2}}\left(\cos \frac{\theta}{2}\right)^{J+m}\left(\sin \frac{\theta}{2}\right)^{J-m} e^{i(J-m) \phi}|J m\rangle .
\end{aligned}
$$

The Bloch coherent states have the following properties:

(I) $\left\langle\Omega_{1} \mid \Omega_{2}\right\rangle=\left(\cos \frac{\theta_{1}}{2} \cos \frac{\theta_{2}}{2}+e^{i\left(\phi_{2}-\phi_{1}\right)} \sin \frac{\theta_{1}}{2} \sin \frac{\theta_{2}}{2}\right)^{2 J}$,

(II) $\frac{2 J+1}{4 \pi} \int_{S^{2}} d \Omega|\Omega\rangle\langle\Omega|=1 \quad$ (resolution of identity),

\footnotetext{
${ }^{9}$ See also $[32,33$ for the description of fuzzy sphere using coherent states.
} 
(III) $y^{\mu} L_{\mu}|\Omega\rangle=J|y||\Omega\rangle$, where $y^{\mu}$ are given by

$$
y^{1}=|y| \sin \theta \cos \phi, \quad y^{2}=|y| \sin \theta \sin \phi, \quad y^{3}=|y| \cos \theta,
$$

(IV) $|\Omega\rangle$ is the ground state of the Hamiltonian,

$$
H(y):=\frac{1}{2}\left(\frac{1}{\sqrt{J(J+1)}} L_{\mu}-y_{\mu}\right)^{2},
$$

(V) $|\Omega\rangle$ minimizes $\sum_{\mu=1}^{3}\left(\Delta L_{\mu}\right)^{2}$, where $\Delta L_{\mu}$ is the standard deviation of $L_{\mu}$.

The first and second properties directly follow from (B.5). In property (II), the integration measure is defined as $\int d \Omega=\int_{0}^{\pi} d \theta \int_{0}^{2 \pi} d \phi \sin \theta$. The remaining properties follow from the fact that the unitary operator $R(\Omega):=e^{\frac{1}{2} \theta e^{i \phi} L_{-}-\frac{1}{2} \theta e^{-i \phi} L_{+}}$in Eq. (B.3) satisfies $R(\Omega) L_{3} R^{-1}(\Omega)=\frac{y^{\mu} L_{\mu}}{|y|}$.

\section{Derivation of equation (3.4)}

In this Appendix, we derive (3.4). As a preliminary step, we first consider the following object:

$$
\left\langle 0, y_{1}|H(y)| 0, y_{2}\right\rangle=\left(E_{0}\left(y_{2}\right)+\frac{1}{2}\left(y_{2}^{\mu}-y^{\mu}\right)^{2}\right)\left\langle 0, y_{1} \mid 0, y_{2}\right\rangle+\left(y_{2}^{\mu}-y^{\mu}\right)\left\langle 0, y_{1}\left|X_{\mu}-y_{2 \mu}\right| 0, y_{2}\right\rangle
$$

From (2.17), we obtain

$$
\lim _{N \rightarrow \infty}\left(y_{2}^{\mu}-y_{1}^{\mu}\right)\left\langle 0, y_{1} \mid 0, y_{2}\right\rangle=0 \quad\left(y_{1}, y_{2} \in \mathcal{M}\right) .
$$

This implies that the coherent states at different points are orthogonal to each other:

$$
\lim _{N \rightarrow \infty}\left\langle 0, y_{1} \mid 0, y_{2}\right\rangle=\delta^{(D)}\left(y_{1}-y_{2}\right) \quad\left(y_{1}, y_{2} \in \mathcal{M}\right)
$$

By applying (2.17) and (C.3) to the object (C.1), we obtain

$$
\lim _{N \rightarrow \infty}\left\langle 0, y_{1}|H(y)| 0, y_{2}\right\rangle=\frac{1}{2}\left(y_{2}^{\mu}-y^{\mu}\right)^{2} \delta^{(D)}\left(y_{1}-y_{2}\right) \quad\left(y_{1}, y_{2} \in \mathcal{M}\right) .
$$

Now, let us compute

$$
f\left(y+\epsilon_{\perp}\right)=\lim _{N \rightarrow \infty} \min _{|\alpha\rangle \in \mathcal{H}^{*}}\left\langle\alpha\left|H\left(y+\epsilon_{\perp}\right)\right| \alpha\right\rangle
$$


where $y \in \mathcal{M}$ and $\epsilon_{\perp}$ is a normal vector. Let us denote by $\mathcal{H}_{\text {coh }}$ the Hilbert space spanned by all the coherent states:

$$
\mathcal{H}_{\mathrm{coh}}=\operatorname{span}\left\{\left|0, y^{\prime}\right\rangle \mid y^{\prime} \in \mathcal{M}\right\}
$$

The total Hilbert space can be written as

$$
\mathcal{H}=\mathcal{H}_{\text {coh }} \oplus \tilde{\mathcal{H}}
$$

By definition, $\tilde{\mathcal{H}}$ is the Hilbert space which does not have any coherent state. We can show that when $\left|\epsilon_{\perp}\right|$ is sufficiently small, the minimum in (C.5) is saturated by an element in $\mathcal{H}_{\text {coh. }}$. Hence, in the computation of ( $(\mathbf{C . 5})$, it suffices to consider the case where $|\alpha\rangle$ is an element of $\mathcal{H}_{\text {coh }}$. Any element $|\alpha\rangle \in \mathcal{H}_{\text {coh }}$ can be expanded by the coherent states as

$$
|\alpha\rangle=\int d^{D} y^{\prime} \alpha\left(y^{\prime}\right)\left|0, y^{\prime}\right\rangle
$$

where $\alpha\left(y^{\prime}\right)$ is a function which vanishes unless $y^{\prime} \in \mathcal{M}$. Since $|\alpha\rangle$ in (C.5) is normalized as $\langle\alpha \mid \alpha\rangle=1$, this gives a constraint on $\alpha\left(y^{\prime}\right)$. By substituting the expansion (C.8) into (C.5) and using (C.4), we obtain

$$
f\left(y+\epsilon_{\perp}\right)=\frac{1}{2} \int d^{D} y^{\prime}\left|\tilde{\alpha}\left(y^{\prime}\right)\right|^{2}\left(y^{\prime \mu}-y^{\mu}-\epsilon_{\perp}^{\mu}\right)^{2} .
$$

Here, $\tilde{\alpha}\left(y^{\prime}\right)$ is the large- $N$ limit of the function which saturates the minimum in (C.5). It satisfies $\int d^{D} y^{\prime}\left|\tilde{\alpha}\left(y^{\prime}\right)\right|^{2}=1$ and vanishes on the outside of $\mathcal{M}$. Obviously, the quantity on the right-hand side of (C.9) is minimized when $\tilde{\alpha}\left(y^{\prime}\right)$ localizes at $y$. Thus, we finally obtain (3.4).

\section{Jacobi identity}

In this Appendix, we show that $W^{\mu \nu}(y)$ defined in (3.16) satisfies

$$
W^{\mu \nu}(y) \partial_{\mu} W^{\rho \sigma}(y)+W^{\mu \rho}(y) \partial_{\mu} W^{\sigma \nu}(y)+W^{\mu \sigma}(y) \partial_{\mu} W^{\nu \rho}(y)=0
$$

This is equivalent to the Jacobi identity for the Poisson bracket defined by $\{A, B\}=$ $W^{\mu \nu}\left(\partial_{\mu} A\right)\left(\partial_{\nu} B\right)$. We consider an arbitrary polynomial $\Phi(X)$ and define a corresponding function by

$$
\phi(y)=\lim _{N \rightarrow \infty}\langle 0, y|\Phi(X)| 0, y\rangle
$$


We can show that

$$
\lim _{N \rightarrow \infty} c\left\langle 0, y\left|\left[X^{\mu}, \Phi(X)\right]\right| 0, y\right\rangle=W^{\mu \nu}(y) \partial_{\nu} \phi(y) .
$$

If we put $\Phi=c\left[X^{\mu}, X^{\nu}\right]$ in the above equations, (D.1) immediately follows from the Jacobi identity of the matrix commutators.

The relation (D.3) can be shown as follows. The right-hand side of (D.3) is the large- $N$ limit of

$$
c\left\langle 0, y\left|\left[X^{\mu}, X^{\nu}\right]\right| 0, y\right\rangle \partial_{\nu}\langle 0, y|\Phi(X)| 0, y\rangle .
$$

The derivative of $|0, y\rangle$ can be read off from the formula in the perturbation theory as

$$
\partial_{\nu}|0, y\rangle=\sum_{n \neq 0} \frac{|n, y\rangle\left\langle n, y\left|X_{\nu}\right| 0, y\right\rangle}{E_{n}(y)-E_{0}(y)} .
$$

By substituting this, and using (3.17), we can see that the large- $N$ limit of (D.4) is equal to the left-hand side of (D.3).

\section{E Classical geometry for fuzzy torus}

In this Appendix, we show that the function $f(y)$ associated with the Hamiltonian (4.12) is given by (4.13). First, note the following inequalities,

$$
\begin{aligned}
E_{0}(y) & =\min _{|\psi\rangle \in \mathcal{H}^{*}}\langle\psi|H(y)| \psi\rangle \\
& \geq \frac{1}{2} \min _{|\psi\rangle \in \mathcal{H}^{*}}\left\langle\psi\left|(U-z)\left(U^{\dagger}-\bar{z}\right)\right| \psi\right\rangle+\frac{1}{2} \min _{|\psi\rangle \in \mathcal{H}^{*}}\left\langle\psi\left|(V-w)\left(V^{\dagger}-\bar{w}\right)\right| \psi\right\rangle \\
& \geq \frac{1}{2}(1-|z|)^{2}+\frac{1}{2}(1-|w|)^{2} .
\end{aligned}
$$

The last inequality follows from the fact that the spectrum of any unitary matrix is contained in the unit circle on the complex plane. On the other hand, for any state vector $|\alpha\rangle \in \mathcal{H}^{*}$, we have

$$
E_{0}(y) \leq\langle\alpha|H(y)| \alpha\rangle .
$$

Hence, if there exists a state vector $|\alpha\rangle \in \mathcal{H}^{*}$ which satisfies

$$
\lim _{N \rightarrow \infty}\langle\alpha|H(y)| \alpha\rangle=\frac{1}{2}(1-|z|)^{2}+\frac{1}{2}(1-|w|)^{2},
$$


we can prove (4.13) from the two inequalities (E.1) and (E.2). In the following, we explicitly construct a state $|\alpha\rangle$ which satisfies (E.3).

We introduce the basis used in Eq. (4.10),

$$
U|m\rangle=e^{i \theta}|m\rangle, \quad V|m\rangle=|m+1\rangle .
$$

For convenience, we extend the range of $m$ to a set of all integers by assuming the periodicity condition $|m+N\rangle=|m\rangle$. With this notation, we introduce a state vector

$$
|\alpha\rangle:=\tilde{c} \sum_{m=-\left[\frac{N-1}{2}\right]}^{\left[\frac{N}{2}\right]} e^{-\frac{a}{2}\left(\frac{m}{N}-\frac{\arg z}{2 \pi}\right)^{2}} e^{-i(\arg w) m}|m\rangle,
$$

where $a$ is a constant of $\mathcal{O}(\sqrt{N}), \tilde{c}$ is a normalization constant determined by $\langle\alpha \mid \alpha\rangle=1$ and $[x]$ stands for the floor function defined by $[x]=\max \{m \in \mathbf{Z} \mid m \leq x\}$. In the large- $N$ limit, we can evaluate $\tilde{c}$ as

$$
\tilde{c}^{-2}=\sum_{m=-\left[\frac{N-1}{2}\right]}^{\left[\frac{N}{2}\right]} e^{-a\left(\frac{m}{N}-\frac{\arg z}{2 \pi}\right)^{2}} \sim N \int_{-\infty}^{\infty} d x e^{-a\left(x-\frac{\arg z}{2 \pi}\right)^{2}}=N \sqrt{\frac{\pi}{a}}
$$

where we have approximated the discrete sum by the integral 10. By repeating similar calculations, we obtain

$$
\lim _{N \rightarrow \infty}\langle\alpha|U| \alpha\rangle=e^{i \arg z}, \quad \lim _{N \rightarrow \infty}\langle\alpha|V| \alpha\rangle=e^{i \arg w}
$$

This implies that $|\alpha\rangle$ satisfies (E.3).

\section{F Derivation of equation (5.7)}

In this Appendix, we derive (5.7). Up to the second order of the perturbation, the ground state energy is given by

$$
E_{0,0}(y)=\frac{\theta+\theta^{\prime}}{2}+\frac{1}{2} \sum_{I=5}^{D}\left(y_{I}\right)^{2}+\left\langle 0,0, y\left|H_{1}(y)+H_{2}(y)\right| 0,0, y\right\rangle
$$

\footnotetext{
${ }^{10}$ The integration range is naively given by $[-1 / 2,1 / 2]$. However, since we assumed that $a=\mathcal{O}(\sqrt{N})$, the main contribution comes only from the neighborhood of the origin. Hence we can extend the range to $[-\infty, \infty]$
} 


$$
+\sum_{(m, n) \neq(0,0)} \frac{\left\langle 0,0, y\left|H_{1}(y)\right| m, n, y\right\rangle\left\langle m, n, y\left|H_{1}(y)\right| 0,0, y\right\rangle}{E_{0,0}^{(0)}(y)-E_{m, n}^{(0)}(y)} .
$$

We first construct the wave functions for the zeroth-order Hamiltonian $H_{0}(y)$ in (5.5) . Since $H_{0}(y)$ is just the sum of two independent (shifted) harmonic oscillators, the wave function is given by the product of wave functions for each oscillator,

$$
\Phi_{m, n}(x, \tilde{x} ; y)=\Psi_{m}^{(\theta)}\left(x ; y^{1}, y^{2}\right) \Psi_{n}^{\left(\theta^{\prime}\right)}\left(\tilde{x} ; y^{3}, y^{4}\right) .
$$

$\Psi_{m}^{(\theta)}\left(x ; y^{1}, y^{2}\right)$ is the wave function of the $m$ th excited state of the shifted harmonic oscillator, given by

$$
\Psi_{m}^{(\theta)}\left(x ; y^{1}, y^{2}\right)=c_{n} h_{n}\left(\frac{x-y_{1}}{\sqrt{\theta}}\right) e^{-\frac{1}{2 \theta}\left(x-y^{1}\right)^{2}+\frac{i y^{2} x}{\theta}},
$$

where $c_{n}=1 / \sqrt{2^{n} n ! \sqrt{\theta \pi}}$ and $h_{n}(\xi)$ is the Hermite polynomial defined by $h_{n}(\xi)=$ $(-1)^{n} e^{\xi^{2}}\left(\frac{d}{d \xi}\right)^{n} e^{-\xi^{2}}$. The operators $\hat{q}^{A}$ act on $\Phi_{m, n}(x, \tilde{x} ; y)$ as follows:

$$
\begin{aligned}
& \left(\hat{q}^{1} \Phi_{m, n}\right)(x, \tilde{x} ; y)=x \Phi_{m, n}(x, \tilde{x} ; y), \\
& \left(\hat{q}^{2} \Phi_{m, n}\right)(x, \tilde{x} ; y)=-i \theta \frac{\partial}{\partial x} \Phi_{m, n}(x, \tilde{x} ; y), \\
& \left(\hat{q}^{3} \Phi_{m, n}\right)(x, \tilde{x} ; y)=\tilde{x} \Phi_{m, n}(x, \tilde{x} ; y), \\
& \left(\hat{q}^{4} \Phi_{m, n}\right)(x, \tilde{x} ; y)=-i \theta^{\prime} \frac{\partial}{\partial \tilde{x}} \Phi_{m, n}(x, \tilde{x} ; y) .
\end{aligned}
$$

By using the wave functions (F.2), we can obtain

$$
\begin{aligned}
& \left\langle 0,0, y\left|e^{i k \cdot \hat{q}}\right| m, n, y\right\rangle=\frac{c_{m} c_{n}}{c_{0}^{2}}\left(i \sqrt{\theta}\left(k_{1}-i k_{2}\right)\right)^{m}\left(i \sqrt{\theta^{\prime}}\left(k_{3}-i k_{4}\right)\right)^{n} e^{-\frac{\theta}{4} k_{a}^{2}-\frac{\theta^{\prime}}{4} k_{\alpha}^{2}+i k \cdot y}, \\
& \left\langle 0,0, y\left|\left\{\hat{q}_{a}-y_{a}, e^{i k \cdot \hat{q}}\right\}\right| m, n, y\right\rangle=i\left[k_{a} \theta-\frac{2 m \chi_{a}}{k_{1}-i k_{2}}\right]\left\langle 0,0, y\left|e^{i k \cdot \hat{q}}\right| m, n, y\right\rangle, \\
& \left\langle 0,0, y\left|\left\{\hat{q}_{\alpha}-y_{\alpha}, e^{i k \cdot \hat{q}}\right\}\right| m, n, y\right\rangle=i\left[k_{\alpha} \theta^{\prime}-\frac{2 n \chi_{\alpha}}{k_{3}-i k_{4}}\right]\left\langle 0,0, y\left|e^{i k \cdot \hat{q}}\right| m, n, y\right\rangle,
\end{aligned}
$$

where $\chi_{a}$ and $\chi_{\alpha}$ are constants given by $\chi_{1}=\chi_{3}=1, \chi_{2}=\chi_{4}=-i$. By using (F.5), we can compute the correction terms in (F.1). In the classical limit where $\theta, \theta^{\prime} \rightarrow 0$, the leading behaviors are given as follows:

$$
\begin{aligned}
& \left\langle 0,0, y\left|H_{1}(y)\right| 0,0, y\right\rangle \sim-y^{I} \phi_{I}(y), \\
& \frac{1}{\sqrt{\theta}}\left\langle 0,0, y\left|H_{1}(y)\right| 1,0, y\right\rangle \sim \frac{1}{\sqrt{2}}\left\{\phi_{1}(y)-i \phi_{2}(y)-y^{I}\left(\partial_{1} \phi_{I}(y)-i \partial_{2} \phi_{I}(y)\right)\right\},
\end{aligned}
$$




$$
\begin{aligned}
& \frac{1}{\sqrt{\theta^{\prime}}}\left\langle 0,0, y\left|H_{1}(y)\right| 0,1, y\right\rangle \sim \frac{1}{\sqrt{2}}\left\{\phi_{3}(y)-i \phi_{4}(y)-y^{I}\left(\partial_{3} \phi_{I}(y)-i \partial_{4} \phi_{I}(y)\right)\right\} \\
& \left\langle 0,0, y\left|H_{2}(y)\right| 0,0, y\right\rangle \sim \frac{1}{2}\left(\phi^{\mu}(y)\right)^{2}
\end{aligned}
$$

In deriving these, the following approximation formula for the delta function is useful:

$$
\int \frac{d^{4} k}{(2 \pi)^{4}} e^{-\frac{\theta}{4} k_{a}^{2}-\frac{\theta^{\prime}}{4} k_{\alpha}^{2}+i k \cdot(y-x)}=\frac{1}{\pi^{2} \theta \theta^{\prime}} e^{-\frac{1}{\theta}\left(y_{a}-x_{a}\right)^{2}-\frac{1}{\theta^{\prime}}\left(y_{\alpha}-x_{\alpha}\right)^{2}} \sim \delta^{(4)}(x-y) .
$$

By substituting (F.6) into (F.1), we finally obtain (5.7).

\section{References}

[1] T. Banks, W. Fischler, S. H. Shenker and L. Susskind, Phys. Rev. D 55, 5112 (1997).

[2] N. Ishibashi, H. Kawai, Y. Kitazawa and A. Tsuchiya, Nucl. Phys. B 498, 467 (1997).

[3] B. de Wit, J. Hoppe and H. Nicolai, Nucl. Phys. B 305, 545 (1988).

[4] M. Hanada, H. Kawai and Y. Kimura, Prog. Theor. Phys. 114, 1295 (2006).

[5] J. Madore, Class. Quant. Grav. 9, 69 (1992).

[6] A. Connes, M. R. Douglas and A. S. Schwarz, JHEP 9802, 003 (1998).

[7] A. P. Balachandran, B. P. Dolan, J. H. Lee, X. Martin and D. O'Connor, J. Geom. Phys. 43, 184 (2002).

[8] M. M. Sheikh-Jabbari and M. Torabian, JHEP 0504, 001 (2005).

[9] J. Arnlind, M. Bordemann, L. Hofer, J. Hoppe and H. Shimada, JHEP 0906, 047 (2009).

[10] H. Shimada, Nucl. Phys. B 685, 297 (2004).

[11] T. Hotta, J. Nishimura and A. Tsuchiya, Nucl. Phys. B 545, 543 (1999).

[12] T. Azeyanagi, M. Hanada, T. Hirata and H. Shimada, JHEP 0903, 121 (2009).

[13] J. Arnlind, J. Hoppe and G. Huisken, J. Diff. Geom. 91, 1 (2012). 
[14] D. Berenstein and E. Dzienkowski, Phys. Rev. D 86, 086001 (2012).

[15] D. Berenstein and E. Dzienkowski, arXiv:1311.1168 [hep-th].

[16] H. Aoki, N. Ishibashi, S. Iso, H. Kawai, Y. Kitazawa and T. Tada, Nucl. Phys. B 565, $176(2000)$.

[17] D. E. Berenstein, M. Hanada and S. A. Hartnoll, JHEP 0902, 010 (2009).

[18] T. Eguchi and H. Kawai, Phys. Rev. Lett. 48, 1063 (1982).

[19] G. Bhanot, U. M. Heller and H. Neuberger, Phys. Lett. B 113, 47 (1982).

[20] G. Parisi, Phys. Lett. B 112, 463 (1982).

[21] D. J. Gross and Y. Kitazawa, Nucl. Phys. B 206, 440 (1982).

[22] R. C. Myers, JHEP 9912, 022 (1999).

[23] H. Steinacker, JHEP 0712, 049 (2007).

[24] S. W. Kim, J. Nishimura and A. Tsuchiya, Phys. Rev. Lett. 108, 011601 (2012).

[25] J. M. Maldacena, Int. J. Theor. Phys. 38, 1113 (1999).

[26] D. Berenstein, JHEP 0601, 125 (2006).

[27] Y. Asano, G. Ishiki and S. Shimasaki, JHEP 1409, 137 (2014).

[28] Y. Asano, G. Ishiki, T. Okada and S. Shimasaki, JHEP 1405, 075 (2014).

[29] S. Catterall and T. Wiseman, JHEP 0712, 104 (2007).

[30] K. N. Anagnostopoulos, M. Hanada, J. Nishimura and S. Takeuchi, Phys. Rev. Lett. 100, 021601 (2008).

[31] J. P. Gazeau, "Coherent states in quantum physics," Weinheim, Germany: WileyVCH (2009).

[32] H. Grosse and P. Presnajder, Lett. Math. Phys. 33, 171 (1995). 
[33] A. B. Hammou, M. Lagraa and M. M. Sheikh-Jabbari, Phys. Rev. D 66, 025025 (2002). 